\title{
SOME INVERSE FRACTIONAL LEGENDRE TRANSFORMS OF GAMMA FUNCTION FORM
}

\author{
AlireZA AnSARI
}

\begin{abstract}
In this paper, using the asymptotic expansion of the ratio of gamma functions, we obtain new inversion formulas for the fractional Legendre transforms. These formulas are given in terms of some integral representations of the inverse Mellin transforms. Also, the closed forms of solutions of the fractional Laplace and Helmholtz equations are obtained by these inversion formulas.
\end{abstract}

\section{Introduction}

The Legendre transform and its inversion formula, for the first time was introduced by Tranter [26]

$$
\begin{aligned}
& \mathscr{T}_{l}\{f(x) ; n\}=\bar{f}_{n}=\int_{-1}^{1} f(x) p_{n}(x) d x, \\
& \mathscr{T}_{l}^{-1}\left\{\bar{f}_{n} ; x\right\}=f(x)=\sum_{n=0}^{\infty}\left(n+\frac{1}{2}\right) \bar{f}_{n} p_{n}(x),
\end{aligned}
$$

where the Legendre polynomials $p_{n}(x)=\frac{(-1)^{n}}{2^{n} n !} \frac{d^{n}}{d x^{n}}\left(1-x^{2}\right)^{n}$ satisfy the Legendre differential equation

$$
\frac{d}{d x}\left(1-x^{2}\right) \frac{d}{d x} y+n(n+1) y=0, \quad n \in \mathbf{N} .
$$

The most important applications of this transform are found in the boundary value problems in spherical coordinates particularly elliptic equations. For example, for the analysis of Laplace, Poisson and Helmoltz equations in spherical coordinates and corresponding operational calculus, see [10-12], [14], [25]. As

Mathematics Subject Classification. Primary 26A33; Secondary 44A20.

Key words and phrases. Fractional Legendre transforms, Mellin transform, Laplace equation, Helmholtz equation.

Received June 10, 2014; revised January 6, 2015. 
generalization of the Legendre differential equation in the sense of fractional derivatives, Klimek and Agrawal introduced a fractional Legendre differential equation with respect to the Riemann-Liouville and Caputo fractional derivatives [18]

$$
{ }^{C} D_{1^{-}}^{\alpha}\left(1-x^{2}\right)^{\alpha} D_{-1^{+}}^{\alpha} y-\frac{\Gamma(n+\alpha+1)}{\Gamma(n+1-\alpha)} y=0, \quad m-1<\alpha \leq m, m \in \mathbf{N} .
$$

To express the properties of this equation, we can refer to the real eigenvalues, orthogonal eigenfunctions and the Legendre polynomials $p_{n}(x)$ as the solution of it. For more details in these properties and similar fractional Sturm-Liouville problems, see [3], [8], [17, 18], [23]. In this sense, for the fractional Legendre equation (1-4) they introduced the following finite fractional Legendre transform with its inversion formula

$$
\begin{aligned}
\mathscr{T}_{\alpha, l}\{f(x) ; n\} & =\bar{f}_{n}=\int_{-1}^{1} f(x) p_{n}(x) d x, \\
\mathscr{T}_{\alpha, l}^{-1}\left\{\bar{f}_{n} ; x\right\} & =f(x)=\sum_{n=0}^{\infty}\left(n+\frac{1}{2}\right) \bar{f}_{n} p_{n}(x) .
\end{aligned}
$$

One of the important property of this transform is the impact on the fractional operator $\mathscr{L}_{\alpha, x}={ }^{C} D_{1^{-}}^{\alpha}\left(1-x^{2}\right)^{\alpha} D_{-1^{+}}^{\alpha}$, which leads to the eigenvalues with respect to the gamma function

$$
\mathscr{T}_{\alpha, l}\left\{{ }^{C} D_{1^{-}}^{\alpha}\left(\left(1-x^{2}\right)^{\alpha} D_{-1^{+}}^{\alpha} f\right)(x) ; n\right\}=\frac{\Gamma(n+\alpha+1)}{\Gamma(n+1-\alpha)} \bar{f}_{n} .
$$

For more applications of this transform in boundary value problems, see [3], [18].

Now, in this paper we intend to survey some partial fractional differential equations which lead to the fractional Legendre equation (1-4) and then, we state new theorems for the inverse fractional Legendre transforms in terms of the gamma function and its approximations. For this purpose, in Section 2 we recall some definitions and lemmas in fractional calculus, operational calculus of the Mellin transform and asymptotic expansion of the gamma function. In Section 3 , we state new theorems for the inverse fractional Legendre transforms with respect to the first, second, third and fourth approximations of the ratio of gamma functions. In Section 4 and Section 5, we use these theorems for applications in the fractional Laplace and Helmoltz equations and we find closed forms of solutions of these equations. Finally, the main conclusions are set.

\section{Preliminaries}

Definition 2.1. For $n-1<\alpha<n, n \in \mathbf{N}$ and $f \in L_{1}(a, b)$, the left-sided and right-sided Riemann-Liouville and Caputo fractional integrals and derivatives are defined as $[16,24]$ 


$$
\begin{aligned}
\left(I_{a^{+}}^{\alpha} f\right)(x) & =\frac{1}{\Gamma(\alpha)} \int_{a}^{x}(x-s)^{\alpha-1} f(s) d s, \quad x>a, \\
\left(I_{b^{-}}^{\alpha} f\right)(x) & =\frac{1}{\Gamma(\alpha)} \int_{x}^{b}(s-x)^{\alpha-1} f(s) d s, \quad x<b, \\
\left(D_{a^{+}}^{\alpha} f\right)(x) & =D^{n}\left(I_{a^{+}}^{n-\alpha} f\right)(x), \\
\left(D_{b^{-}}^{\alpha} f\right)(x) & =(-D)^{n}\left(I_{b^{-}}^{n-\alpha} f\right)(x), \\
\left({ }^{C} D_{a^{+}}^{\alpha} f\right)(x) & =\left(I_{a^{+}}^{n-\alpha} D^{n} f\right)(x), \\
\left({ }^{C} D_{b^{-}}^{\alpha} f\right)(x) & =\left(I_{b^{-}}^{n-\alpha}(-D)^{n} f\right)(x),
\end{aligned}
$$

where $D=\frac{d}{d x}$.

LEMma 2.2 [7]. Let $\Phi(s)$ be an entire function such that for even and odd functions $u(r)$ and $v(r)$, we have $\phi( \pm i r)=u(r) \pm i v(r)$. Then for the $M$-Wright function $[20,22]$

$$
M_{\alpha}(x)=\sum_{k=0}^{\infty} \frac{(-x)^{k}}{k ! \Gamma(-\alpha k+1-\alpha)}, \quad 0<\alpha<1,
$$

the following relations hold for $c_{1}<\Re(s)<c_{2}$

$$
\begin{aligned}
e^{-\lambda \Phi(s)} & =\int_{-\infty}^{\infty} e^{s \xi} \mathscr{A}(\xi, \lambda) d \xi \\
e^{-\lambda \Phi\left(s^{\alpha}\right)} & =\int_{-\infty}^{\infty} e^{s \xi} \mathscr{A}_{\alpha}(\xi, \lambda) d \xi,
\end{aligned}
$$

where the functions $\mathscr{A}_{\alpha}(\xi, \lambda)$ and $\mathscr{A}(\xi, \lambda)$ are shown by

$$
\begin{gathered}
\mathscr{A}_{\alpha}(\xi, \lambda)=-\frac{\alpha}{\pi \xi^{\alpha+1}} \int_{-\infty}^{\infty} \tau \mathscr{A}(\tau, \lambda) M_{\alpha}\left(\tau(-\xi)^{-\alpha}\right) d \tau, \\
\mathscr{A}(\xi, \lambda)=\frac{1}{\pi} \int_{0}^{\infty} e^{-\lambda u(r)} \cos (r \xi+\lambda v(r)) d r .
\end{gathered}
$$

Lemma 2.3. In special case $\Phi(s)=s$, we can write the following integral representation for $e^{-\lambda s^{\alpha}}$ in terms of the M-Wright function as [4-6]

$$
e^{-\lambda s^{\alpha}}=\alpha \lambda \int_{0}^{\infty} \frac{1}{t^{\alpha+1}} M_{\alpha}\left(\lambda t^{-\alpha}\right) e^{-s t} d t, \quad 0<\alpha<1,
$$



and for $\alpha=\frac{1}{2}$ it gives rise to

$$
e^{-\lambda \sqrt{s}}=\frac{\lambda}{\sqrt{\pi}} \int_{0}^{\infty} \frac{1}{t \sqrt{t}} e^{-\lambda^{2} / 4 t} e^{-s t} d t
$$

LEMma 2.4. Asymptotic formula for the ratio of gamma functions is given by the following series in terms of the Nörlund's generalized Bernoulli polynomial $B_{k}^{(a)}(\gamma)[13,15]$

$$
\begin{aligned}
& \frac{\Gamma(n+\gamma)}{\Gamma(n+\beta)}= \sum_{k=0}^{\infty} \frac{(-1)^{k}}{k !} B_{k}^{(\gamma-\beta+1)}(\gamma) \frac{\Gamma(\beta-\gamma+k)}{\Gamma(\beta-\gamma)} \frac{1}{n^{\beta-\gamma+k}} \\
&= \frac{1}{n^{\beta-\gamma}}-\frac{(\beta-\gamma)(\beta+\gamma-1)}{2 n^{\beta-\gamma+1}} \\
&+\frac{\left(\frac{1}{8} \gamma^{2}+\frac{1}{4} \gamma \beta-\frac{7}{24} \gamma-\frac{5}{24} \beta+\frac{1}{12}+\frac{1}{8} \beta^{2}\right)(\beta-\gamma)(\beta+\gamma+1)}{2 n^{\beta-\gamma+2}} \\
&-\frac{\left(\frac{1}{16} \gamma+\frac{1}{48} \beta-\frac{1}{8} \gamma \beta-\frac{1}{24} \beta^{2}+\frac{1}{16} \gamma^{2} \beta+\frac{1}{16} \gamma \beta^{2}-\frac{1}{12} \gamma^{2}\right.}{6 n^{\beta-\gamma+3}} \\
&+\frac{\left.+\frac{1}{48} \gamma^{3}+\frac{1}{48} \beta^{3}\right) \Pi_{j=0}^{2}(\beta-\gamma+j)}{6 n^{\beta-\gamma+3}} \\
&+\frac{\left(-\frac{1}{320} \gamma+\frac{1}{320} \beta+\frac{11}{576} \gamma \beta+\frac{1}{1152} \beta^{2}-\frac{7}{192} \gamma^{2} \beta-\frac{5}{192} \gamma \beta^{2}\right.}{24 n^{\beta-\gamma+4}} \\
&+\frac{+\frac{25}{1152} \gamma^{2}-\frac{1}{64} \gamma^{3}+\frac{1}{384} \gamma^{4}-\frac{1}{192} \beta^{3}}{24 n^{\beta-\gamma+4}} \\
&+\frac{\left.-\frac{1}{720}+\frac{1}{96} \gamma^{3} \beta+\frac{1}{64} \gamma^{2} \beta^{2}+\frac{1}{96} \gamma \beta^{3}+\frac{1}{384} \beta^{4}\right) \Pi_{j=0}^{3}(\beta-\gamma+j)}{24 n^{\beta-\gamma+4}} \\
&-\frac{\left(-\frac{1}{1152} \gamma-\frac{1}{1920} \beta+\frac{1}{1152} \gamma \beta+\frac{13}{11520} \beta^{2}+\frac{17}{2304} \gamma^{2} \beta+\frac{5}{2304} \gamma \beta^{2}\right.}{120 n^{\beta-\gamma+5}} \\
&+\frac{-\frac{23}{11520} \gamma^{2}+\frac{11}{2304} \gamma^{3}-\frac{5}{2304} \gamma^{4}-\frac{1}{2304} \beta^{3}+\frac{1}{3840} \gamma^{5}-\frac{1}{144} \gamma^{3} \beta}{120 n^{\beta-\gamma+5}} \\
&+\frac{-\frac{1}{128} \gamma^{2} \beta^{2}-\frac{1}{288} \gamma \beta^{3}+\frac{1}{768} \gamma^{4} \beta+\frac{1}{384} \gamma^{3} \beta^{2}+\frac{1}{384} \gamma^{2} \beta^{3}}{120 n^{\beta-\gamma+5}} \\
&\left.+\frac{1}{768} \gamma \beta^{4}-\frac{1}{2304} \beta^{4}+\frac{1}{3840} \beta^{5}\right) \Pi_{j=0}^{4}(\beta-\gamma+5 \\
& 120 n^{\beta-5} \\
&+
\end{aligned}
$$

and in special case $\gamma=1+\alpha, \beta=1-\alpha$, it follows that

$$
\begin{aligned}
& \frac{\Gamma(n+1+\alpha)}{\Gamma(n+1-\alpha)} \\
& \quad=n^{2 \alpha}\left(1+\frac{\alpha}{n}-\frac{\alpha(2 \alpha-1)(\alpha-1)}{12 n^{2}}-\frac{\alpha^{2}(2 \alpha-1)(2 \alpha-2)}{72 n^{3}}+O\left(n^{-4}\right)\right) .
\end{aligned}
$$


Example 2.5. According to the relation (2-12), for the first approximation of the above asymptotic expansion, we have

$$
e^{-\lambda s^{2 \alpha}}=2 \alpha \lambda \int_{0}^{\infty} \frac{1}{t^{2 \alpha+1}} M_{2 \alpha}\left(\lambda t^{-2 \alpha}\right) e^{-s t} d t .
$$

Example 2.6. In this example, we intend to find the inverse Mellin transform of function $F(s)=e^{-\lambda\left(s^{2 \alpha}+\alpha s^{2 \alpha-1}\right)}$. Since the given function $F$ does not satisfy the relations (2-8) and (2-9), we consider the direct inversion formula

$$
I=\frac{1}{2 \pi i} \int_{c-i \infty}^{c+i \infty} e^{-\lambda\left(s^{2 \alpha}+\alpha s^{2 \alpha-1}\right)} x^{-s} d s .
$$

For the above integral, $s=0$ is a branch point and we can use the well-known Titchmarsh theorem [2], [9]

$$
f(x)=\mathscr{M}^{-1}\{F(s) ; x\}=\frac{1}{\pi} \int_{0}^{\infty} x^{r} \Im\left(F\left(r e^{-i \pi}\right)\right) d r,
$$

to obtain the following integral representation for the second approximation of the asymptotic expansion (2-15)

$$
e^{-\lambda\left(s^{2 \alpha}+\alpha s^{2 \alpha-1}\right)}=\int_{-\infty}^{\infty} e^{s \xi} \mathscr{A}^{(2)}\left(e^{\xi}, \lambda\right) d \xi,
$$

where

$$
\begin{aligned}
\mathscr{A}^{(2)}(x, \lambda)= & \frac{1}{\pi} \int_{0}^{\infty} x^{r} e^{-\lambda\left(r^{2 \alpha} \cos (2 \pi \alpha)+\alpha r^{2 \alpha-1} \cos ((2 \alpha-1) \pi)\right)} \\
& \times \sin \left(\lambda\left(r^{2 \alpha} \sin (2 \pi \alpha)+\alpha r^{2 \alpha-1} \sin ((2 \alpha-1) \pi)\right)\right) d r .
\end{aligned}
$$

Also, according to the relation (2-13) we have the following integral representation

$$
e^{-\lambda \sqrt{1 / 4+s^{2 \alpha}+\alpha s^{2 \alpha-1}}}=\frac{\lambda}{\sqrt{\pi}} \int_{0}^{\infty} \int_{-\infty}^{\infty} \frac{1}{t \sqrt{t}} e^{-\lambda^{2} / 4 t-(1 / 4) t+s \xi} \mathscr{A}^{(2)}\left(e^{\xi}, t\right) d \xi d t
$$

Example 2.7. Using the Titchmarsh theorem (2-18), we can obtain the following integral representations for the third and fourth approximations of the asymptotic expansion (2-15)

$$
\begin{gathered}
e^{-\lambda \sqrt{1 / 4+s^{2 \alpha}+\alpha s^{2 \alpha-1}-\alpha_{1} s^{2 \alpha-2}}}=\frac{\lambda}{\sqrt{\pi}} \int_{0}^{\infty} \int_{-\infty}^{\infty} \frac{1}{t \sqrt{t}} e^{-\lambda^{2} / 4 t-(1 / 4) t+s \xi} \mathscr{A}^{(3)}\left(e^{\xi}, t\right) d \xi d t \\
e^{-\lambda \sqrt{1 / 4+s^{2 \alpha}+\alpha s^{2 \alpha-1}-\alpha_{1} s^{2 \alpha-2}-\alpha_{2} s^{2 \alpha-3}}} \\
=\frac{\lambda}{\sqrt{\pi}} \int_{0}^{\infty} \int_{-\infty}^{\infty} \frac{1}{t \sqrt{t}} e^{-\lambda^{2} / 4 t-(1 / 4) t+s \xi} \mathscr{A}^{(4)}\left(e^{\xi}, t\right) d \xi d t
\end{gathered}
$$


where $\alpha_{1}=\frac{\alpha(2 \alpha-1)(\alpha-1)}{12}, \alpha_{2}=\frac{\alpha^{2}(2 \alpha-1)(2 \alpha-2)}{72}$ and

$$
\begin{aligned}
\mathscr{A}^{(3)}(x, \lambda)= & \frac{1}{\pi} \int_{0}^{\infty} x^{r} e^{\left.-\lambda\left(r^{2 \alpha} \cos (2 \pi \alpha)+\alpha r^{2 \alpha-1} \cos ((2 \alpha-1) \pi)\right)-\alpha_{1} r^{2 \alpha-2} \cos ((2 \alpha-2) \pi)\right)} \\
& \times \sin \left(\lambda\left(r^{2 \alpha} \sin (2 \pi \alpha)+\alpha r^{2 \alpha-1} \sin ((2 \alpha-1) \pi)\right)\right. \\
& \left.\left.\left.\quad-\alpha_{1} r^{2 \alpha-2} \sin ((2 \alpha-2) \pi)\right)\right)\right) d r
\end{aligned}
$$

$$
\begin{aligned}
& \mathscr{A}^{(4)}(x, \lambda) \\
& =\frac{1}{\pi} \int_{0}^{\infty} x^{r} e^{\left.-\lambda\left(r^{2 \alpha} \cos (2 \pi \alpha)+\alpha r^{2 \alpha-1} \cos ((2 \alpha-1) \pi)\right)-\alpha_{1} r^{2 \alpha-2} \cos ((2 \alpha-2) \pi)-\alpha_{2} r^{2 \alpha-3} \cos ((2 \alpha-3) \pi)\right)} \\
& \quad \times \sin \left(\lambda\left(r^{2 \alpha} \sin (2 \pi \alpha)+\alpha r^{2 \alpha-1} \sin ((2 \alpha-1) \pi)\right)\right. \\
& \left.\left.\quad-\sum_{j=1}^{2} \alpha_{j} r^{2 \alpha-j-1} \sin ((2 \alpha-j-1) \pi)\right)\right) d r .
\end{aligned}
$$

\section{Main theorems}

THEOREM 3.1. For the entire function $\Phi(n)$, the inverse fractional Legendre transform of $\bar{f}_{n}=r^{\Phi\left(n^{\alpha}\right)}$ is given by

$$
\mathscr{T}_{\alpha, l}^{-1}\left\{r^{\Phi\left(n^{\alpha}\right)} ; n \rightarrow x\right\}=\frac{1}{2} \int_{-\infty}^{\infty}\left(1-e^{2 \xi}\right)\left(1-2 e^{\xi} x+e^{2 \xi}\right)^{-3 / 2} \mathscr{A}_{\alpha}\left(\xi, \ln \left(\frac{1}{r}\right)\right) d \xi
$$

Proof. If we use the following fact $[14,25]$

$$
\mathscr{T}_{\alpha, l}^{-1}\left\{r^{n} ; n \rightarrow x\right\}=\frac{1}{2}\left(1-r^{2}\right)\left(1-2 r x+r^{2}\right)^{-3 / 2},
$$

and apply the integral representation (2-9), then we get the inverse fractional Legendre transform of $\bar{f}_{n}$ as

$$
\mathscr{T}_{\alpha, l}^{-1}\left\{r^{\Phi\left(n^{\alpha}\right)} ; n \rightarrow x\right\}=\int_{-\infty}^{\infty} \mathscr{T}_{\alpha, l}^{-1}\left\{\left(e^{\xi}\right)^{n} ; n \rightarrow x\right\} \mathscr{A}_{\alpha}\left(\xi, \ln \left(\frac{1}{r}\right)\right) d \xi
$$

or equivalently the relation (3-1).

THEOREM 3.2. For the entire function $\Phi(n)$ and modified Bessel function of order $v, I_{v}$, the inverse fractional Legendre transform of $\bar{f}_{n}=I_{\Phi\left(n^{\alpha}\right)}$ is given by the following formula in terms of the relation (3-1) 


$$
\begin{aligned}
\mathscr{T}_{\alpha, l}^{-1}\left\{I_{\Phi\left(n^{\alpha}\right)}(z) ; n \rightarrow x\right\}= & -\int_{-\infty}^{-1} e^{z u} \frac{1}{\pi\left(u^{2}-1\right)^{1 / 2}} \Im\left[\mathscr{T}_{\alpha, l}^{-1}\left\{A^{\Phi\left(n^{\alpha}\right)} ; n \rightarrow x\right\}\right] d u, \\
& +\int_{-1}^{1} e^{z u} \frac{1}{\pi\left(1-u^{2}\right)^{1 / 2}} \Re\left[\mathscr{T}_{\alpha, l}^{-1}\left\{B^{\Phi\left(n^{\alpha}\right)} ; n \rightarrow x\right\}\right] d u,
\end{aligned}
$$

where $A=\frac{2 e^{i \pi}}{\left[(-u-1)^{1 / 2}+(-u+1)^{1 / 2}\right]^{2}}$ and $B=e^{i \cos ^{-1}(u)}$.

Proof. We use the following fact for the inverse Mellin transform of modified Bessel function [21]

$$
I_{v}(z)=\int_{-\infty}^{\infty} e^{z u} f(u) d u,
$$

where the function $f$ is given in terms of the Heaviside unite step function

$$
\begin{aligned}
f(u)= & -\frac{1}{\pi\left(u^{2}-1\right)^{1 / 2}} \Im\left(\frac{2 e^{i \pi}}{\left[(-u-1)^{1 / 2}+(-u+1)^{1 / 2}\right]^{2}}\right)^{v} H(-1-u) \\
& +\frac{1}{\pi\left(1-u^{2}\right)^{1 / 2}} \Re\left(e^{i \cos ^{-1}(u)}\right)^{v}(H(u+1)-H(u-1)) .
\end{aligned}
$$

The relation (3-4) can be easily derived by applying (3-1) for the inverse fractional Legendre transform of $r^{\Phi\left(n^{\alpha}\right)}$.

THEOREM 3.3. The inverse fractional Legendre transforms of $r^{\sqrt{1 / 4+n^{2 x}}}$ and $r \sqrt{1 / 4+n^{2 \alpha}+\alpha n^{2 \alpha-1}}$ are given by

$$
\begin{aligned}
g_{r}(x)= & \mathscr{T}_{\alpha, l}^{-1}\left\{r^{\sqrt{1 / 4+n^{2 \alpha}}} ; n \rightarrow x\right\} \\
= & -\frac{\alpha \ln (r)}{2 \sqrt{\pi}} \int_{0}^{\infty} \int_{0}^{\infty} \frac{e^{-\ln ^{2}(r) / 4 t-(1 / 4) t}}{\sqrt{t} \xi^{2 \alpha+1}} \\
& \times M_{2 \alpha}\left(t \xi^{-2 \alpha}\right)\left(1-e^{-2 \xi}\right)\left(1-2 e^{-\xi} x+e^{-2 \xi}\right)^{-3 / 2} d \xi d t, \\
\mathscr{T}_{\alpha, l}^{-1}\left\{r \sqrt{1 / 4+n^{2 \alpha}+\alpha n^{2 \alpha-1}} ; n \rightarrow x\right\} & \\
= & -\frac{\ln (r)}{2 \sqrt{\pi}} \int_{0}^{\infty} \int_{-\infty}^{\infty} \frac{1}{t \sqrt{t}} e^{-\ln ^{2}(r) / 4 t-(1 / 4) t}\left(1-e^{2 \xi}\right) \\
& \times\left(1-2 e^{\xi} x+e^{2 \xi}\right)^{-3 / 2} \mathscr{A}^{(2)}\left(e^{\xi}, t\right) d \xi d t,
\end{aligned}
$$

where the function $\mathscr{A}^{(2)}(\xi, t)$ is given by $(2-20)$.

Proof. The results can be easily obtained by applying the relations (2-16), (2-21) and (3-2) simultaneously. 
COROLlaRY 3.4. The inverse fractional Legendre transforms of $I \sqrt{1 / 4+n^{2 x}}(z)$ and $I \sqrt{1 / 4+n^{2 \alpha}+\alpha n^{2 \alpha-1}}(z)$ are obtained by the formula $(3-4)$ in terms of the relations (3-7) and (3-8).

COROLlary 3.5. For the inverse fractional Legendre transforms of expressions

$$
\begin{array}{cl}
r^{\sqrt{1 / 4+n^{2 \alpha}+\alpha n^{2 \alpha-1}-\alpha_{1} n^{2 \alpha-2}}}, & r^{\sqrt{1 / 4+n^{2 \alpha}+\alpha n^{2 \alpha-1}-\alpha_{1} n^{2 \alpha-2}-\alpha_{2} n^{2 \alpha-3}}}, \\
I \sqrt{1 / 4+n^{2 \alpha}+\alpha n^{2 \alpha-1}-\alpha_{1} n^{2 \alpha-2}}(z), & I \sqrt{1 / 4+n^{2 \alpha}+\alpha n^{2 \alpha-1}-\alpha_{1} n^{2 \alpha-2}-\alpha_{2} n^{2 \alpha-3}}(z),
\end{array}
$$

we can write the similar identities in terms of the relations (2-22) and (2-23).

\section{The Laplace equation on the sphere of unit radius}

As an application of the fractional Legendre transform in boundary value problems, we express the following Dirichlet problem within sphere of unit radius

$$
\begin{aligned}
& \frac{1}{r^{2}}\left[\frac{\partial}{\partial r} r^{2} \frac{\partial}{\partial r}+\mathscr{L}_{\alpha, x}\right] \Psi(r, \theta)=0, \quad 0 \leq r \leq 1,0 \leq \theta \leq \pi, \alpha>0, \\
& \Psi(1, \theta)=f(\cos (\theta)), \\
& \Psi(r, \theta) \rightarrow 0, \quad r \rightarrow 0,
\end{aligned}
$$

where

$$
\mathscr{L}_{\alpha, x}={ }^{C} D_{1^{-}}^{\alpha}\left(1-x^{2}\right)^{\alpha} D_{-1^{+}}^{\alpha}, \quad x=\cos (\theta) .
$$

If we consider $\bar{\Psi}_{n}(r)=\mathscr{T}_{\alpha, l}\{\Psi(r, \theta) ; x \rightarrow n\}$, then it follows from relation (1-4) that the function $\bar{\Psi}$ satisfy the Cauchy-Euler differential equation

$$
\left[\frac{\partial}{\partial r} r^{2} \frac{\partial}{\partial r}-\frac{\Gamma(n+\alpha+1)}{\Gamma(n+1-\alpha)}\right] \bar{\Psi}_{n}(r)=0
$$

with boundary condition

$$
\bar{\Psi}(1, n)=\bar{f}_{n}=\mathscr{T}_{\alpha, l}\{f(x) ; x \rightarrow n\} .
$$

Since the solution of the problem tends to 0 when $r \rightarrow 0$, we get the solution of second order differential equation as

$$
\bar{\Psi}_{n}(r)=\bar{f}_{n} r^{-1 / 2+\sqrt{1 / 4+\Gamma(n+\alpha+1) / \Gamma(n+1-\alpha)}},
$$

which implies that

$$
\Psi(r, \theta)=\mathscr{T}_{\alpha, l}^{-1}\left\{\bar{f}_{n} r^{-1 / 2+\sqrt{1 / 4+\Gamma(n+\alpha+1) / \Gamma(n+1-\alpha)}} ; n \rightarrow \cos (\theta)\right\} .
$$


The required solution $\Psi(r, \theta)$ can be obtained by the convolution theorem of Legendre transform of two bounded integrable functions $f$ and $g$, that is [25]

$$
\Psi(r, \theta)=\frac{1}{\pi} \int_{0}^{\pi} \int_{0}^{\pi} f\left(\cos \left(\theta^{\prime}\right)\right) \sin \left(\theta^{\prime}\right) g(\cos (\Theta)) d \theta d \phi,
$$

where $\Theta$ is given by the equation

$$
\cos (\Theta)=\cos (\theta) \cos \left(\theta^{\prime}\right)+\sin (\theta) \sin \left(\theta^{\prime}\right) \cos (\phi),
$$

and $\quad f(x)=\mathscr{T}_{\alpha, l}^{-1}\left\{\bar{f}_{n} ; n \rightarrow \cos (\theta)\right\}, \quad g(x)=\mathscr{T}_{\alpha, l}^{-1}\left\{r^{-1 / 2+\sqrt{1 / 4+\Gamma(n+\alpha+1) / \Gamma(n+1-\alpha)}} ;\right.$ $n \rightarrow \cos (\theta)\}$. To simplify our solution we choice two cases for $0<\alpha<1$. The case $1<\alpha<2$ can be written as the third and fourth approximations with respect to the relations (2-22) and (2-23).
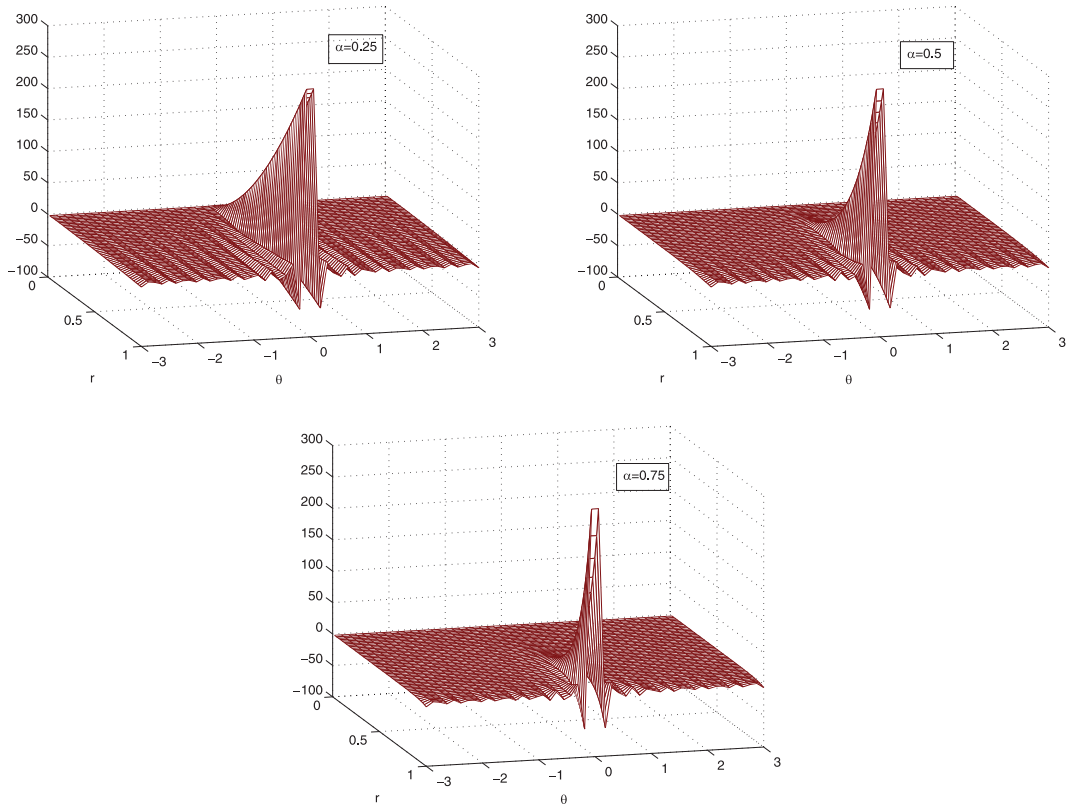

FIGURE 1. The fractional kernel $g_{r}(x)$ for $\alpha=\frac{1}{4}, \alpha=\frac{1}{2}, \alpha=\frac{3}{4}$

4.1. The first approximation for $0<\alpha<\frac{1}{2}$

In this case, by the first approximation of the asymptotic expansion (2-15), we have

$$
\bar{\Psi}_{n}(r)=\bar{f}_{n} r^{-1 / 2+\sqrt{1 / 4+n^{2 \alpha}}},
$$


which by applying the integral (3-7) for $\mathscr{T}_{\alpha, l}^{-1}\left\{r^{-1 / 2+\sqrt{1 / 4+n^{2 \alpha}}} ; n \rightarrow x\right\}$, we get the function $g_{r}(x)$ as follows

$$
\begin{aligned}
g_{r}(x)= & -\frac{\alpha \ln (r)}{2 \sqrt{\pi r}} \int_{0}^{\infty} \int_{0}^{\infty} \frac{e^{-\ln ^{2}(r) / 4 t-(1 / 4) t}}{\sqrt{t} \xi^{2 \alpha+1}} \\
& \times M_{2 \alpha}\left(t \xi^{-2 \alpha}\right)\left(1-e^{-2 \xi}\right)\left(1-2 e^{-\xi} x+e^{-2 \xi}\right)^{-3 / 2} d \xi d t .
\end{aligned}
$$

\subsection{The second approximation for $\frac{1}{2} \leq \alpha<1$}

In this case, by the second approximation of the asymptotic expansion (2-15), we have

$$
\bar{\Psi}_{n}(r)=\bar{f}_{n} r^{-1 / 2+\sqrt{1 / 4+n^{2 \alpha}+\alpha n^{2 \alpha-1}}},
$$

which by applying the integral (3-8) for $\mathscr{T}_{\alpha, l}^{-1}\left\{r^{-1 / 2+\sqrt{1 / 4+n^{2 \alpha}+\alpha n^{2 \alpha-1}}} ; n \rightarrow x\right\}$, we get the function $g_{r}(x)$ as follows

$$
\begin{aligned}
g_{r}(x)= & -\frac{\ln (r)}{2 \sqrt{\pi r}} \int_{0}^{\infty} \int_{-\infty}^{\infty} \frac{1}{t \sqrt{t}} e^{-\ln ^{2}(r) / 4 t-(1 / 4) t} \\
& \times\left(1-e^{2 \xi}\right)\left(1-2 e^{\xi} x+e^{2 \xi}\right)^{-3 / 2} \mathscr{A}^{(2)}\left(e^{\xi}, t\right) d \xi d t .
\end{aligned}
$$

Figure 1 shows the fractional kernel $g_{r}(x)$ for $\alpha=\frac{1}{4}, \alpha=\frac{1}{2}, \alpha=\frac{3}{4}$.

\section{The Helmholtz equation on the sphere of unit radius}

Similar to the previous section for the fractional Legendre transform in boundary value problems, we express the following Helmholtz equation within sphere of unit radius

$$
\begin{aligned}
\frac{1}{r^{2}}\left[\frac{\partial}{\partial r} r^{2} \frac{\partial}{\partial r}+\mathscr{L}_{\alpha, x}\right] \Psi(r, \theta)-\kappa^{2} \Psi(r, \theta) & =0, \quad 0 \leq r \leq 1,0 \leq \theta \leq \pi, \alpha>0, \\
\Psi(1, \theta) & =f(\cos (\theta)) \\
\Psi(r, \theta) \rightarrow 0, & r \rightarrow 0 .
\end{aligned}
$$

By considering $\bar{\Psi}_{n}(r)=\mathscr{T}_{\alpha, l}\{\Psi(r, \theta) ; x \rightarrow n\}$ and relation (1-4), the function $\bar{\Psi}$ satisfy the following differential equation

$$
\left[\frac{\partial}{\partial r} r^{2} \frac{\partial}{\partial r}-\left(r^{2} \kappa^{2}+\frac{\Gamma(n+\alpha+1)}{\Gamma(n+1-\alpha)}\right)\right] \bar{\Psi}_{n}(r)=0,
$$

with boundary condition

$$
\bar{\Psi}(1, n)=\bar{f}_{n}=\mathscr{T}_{\alpha, l}\{f(x) ; x \rightarrow n\} .
$$


Now, by the change of function $\bar{\Theta}_{n}(r)=\sqrt{r} \bar{\Psi}_{n}(r)$, the above equation can be rewritten into the modified Bessel differential equation

$$
\left[r^{2} \frac{\partial^{2}}{\partial r^{2}}+r \frac{\partial}{\partial r}-\left(r^{2} \kappa^{2}+\frac{\Gamma(n+\alpha+1)}{\Gamma(n+1-\alpha)}+\frac{1}{4}\right)\right] \bar{\Theta}_{n}(r)=0,
$$

which has the following solution in terms of the modified Bessel function of first kind $I_{v}$ and second kind $K_{v}$

$$
\bar{\Theta}_{n}(r)=C_{1} I \sqrt{1 / 4+\Gamma(n+\alpha+1) / \Gamma(n+1-\alpha)}(\kappa r)+C_{2} K_{\sqrt{1 / 4+\Gamma(n+\alpha+1) / \Gamma(n+1-\alpha)}}(\kappa r) .
$$

Because of the boundedness of the solution, we must write the above solution as

$$
\bar{\Theta}_{n}(r)=C_{1} I \sqrt{1 / 4+\Gamma(n+\alpha+1) / \Gamma(n+1-\alpha)}(\kappa r),
$$

or equivalently we have

$$
\bar{\Psi}_{n}(r)=C_{1} \frac{1}{\sqrt{r}} I \sqrt{1 / 4+\Gamma(n+\alpha+1) / \Gamma(n+1-\alpha)}(\kappa r) .
$$

To obtain the unknown constant $C_{1}$, by applying the boundary condition we finally get the solution of the Helmholtz equation in the following form

$$
\Psi(r, \theta)=\mathscr{T}_{\alpha, l}^{-1}\left\{\frac{1}{\sqrt{r}} \bar{f}_{n} \frac{I \sqrt{1 / 4+\Gamma(n+\alpha+1) / \Gamma(n+1-\alpha)}(\kappa r)}{I \sqrt{1 / 4+\Gamma(n+\alpha+1) / \Gamma(n+1-\alpha)}(\kappa)} ; n \rightarrow \cos (\theta)\right\},
$$

or equivalently in series form

$$
\Psi(r, \theta)=\frac{1}{\sqrt{r}} \sum_{n=0}^{\infty}\left(n+\frac{1}{2}\right) \bar{f}_{n} \frac{I \sqrt{1 / 4+\Gamma(n+\alpha+1) / \Gamma(n+1-\alpha)}(\kappa r)}{I \sqrt{1 / 4+\Gamma(n+\alpha+1) / \Gamma(n+1-\alpha)}(\kappa)} p_{n}(\cos (\theta)) .
$$

In special case $\bar{f}_{n}=I \sqrt{1 / 4+\Gamma(n+\alpha+1) / \Gamma(n+1-\alpha)}(\kappa)$ and for the first and second approximations, we can get the inverse fractional Legendre transform of $I_{\sqrt{1 / 4+n^{2 \alpha}}}(\kappa r)$ and $I_{\sqrt{1 / 4+n^{2 \alpha}+\alpha n^{2 \alpha-1}}}(\kappa r)$ in terms of the integral representations (3-4), (3-7) and (3-8). The third and fourth approximations can be obtained using the results of Corollary 3.5. Figure 2 shows the inverse fractional

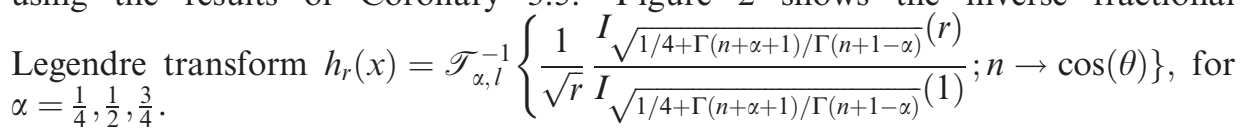

\section{Other coordinates}

Many boundary value problems with fractional derivatives in other coordinates can be solved by this approach. For example, in prolate spheroidal coordinates the Laplace equation takes the form [19] 

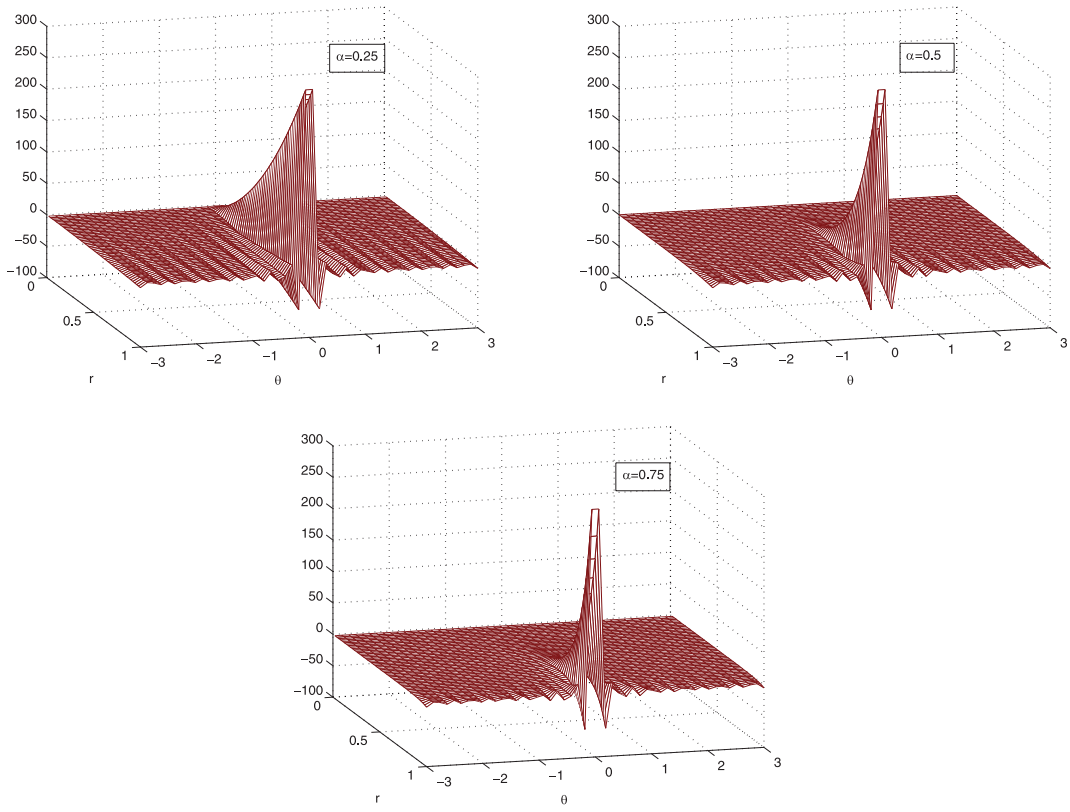

FIGURE 2. The fractional kernel $h_{r}(x)$ for $\alpha=\frac{1}{4}, \alpha=\frac{1}{2}, \alpha=\frac{3}{4}$

$$
\begin{gathered}
{\left[\frac{1}{\sinh (\beta)} \frac{\partial}{\partial \beta} \sinh (\beta) \frac{\partial}{\partial \beta}+\mathscr{L}_{\alpha, x}\right] \Psi(\beta, \theta)=0, \quad 0 \leq \beta<\infty, 0 \leq \theta \leq \theta_{0}, \alpha>0} \\
\Psi\left(\beta_{0}, \theta\right)=f(\cos (\theta)), \\
\Psi(\beta, \theta) \rightarrow 0, \quad \beta \rightarrow 0 .
\end{gathered}
$$

After using the fractional Legendre transform on the equation, we get the Legendre differential equation with a solution in terms of the Toroidal type function [1]

$$
\bar{\Psi}_{n}(r)=C_{1} p_{-1 / 2+1 / 2 \sqrt{1+4(\Gamma(n+\alpha+1) / \Gamma(n+1-\alpha))}}(\cosh (\beta)),
$$

which by applying the boundary conditions we have

$$
\bar{\Psi}_{n}(r)=\bar{f}_{n} \frac{p_{-1 / 2+1 / 2 \sqrt{1+4(\Gamma(n+\alpha+1) / \Gamma(n+1-\alpha))}}(\cosh (\beta))}{p_{-1 / 2+1 / 2 \sqrt{1+4(\Gamma(n+\alpha+1) / \Gamma(n+1-\alpha))}}\left(\cosh \left(\beta_{0}\right)\right)} .
$$

In order to obtain the inversion of the above equation, if we consider the special case $\bar{f}_{n}=p_{-1 / 2+1 / 2 \sqrt{1+4(\Gamma(n+\alpha+1) / \Gamma(n+1-\alpha))}}\left(\cosh \left(\beta_{0}\right)\right)$, then by the following integral representation for the Legendre polynomials $p_{v}$

$$
p_{v}(z)=\frac{1}{\pi} \int_{0}^{\pi}\left(z-\sqrt{z^{2}-1} \cos (t)\right)^{v} d t, \quad \Re(z)>0,
$$


and relations (3-7) and (3-8), we can get the inversions for case $0<\alpha<1$ as the first and second approximations

$$
\begin{aligned}
& \mathscr{T}_{\alpha, l}^{-1}\left\{p_{-1 / 2+\sqrt{1 / 4+n^{2 \alpha}}}(\cosh (\beta)) ; n \rightarrow x\right\} \\
& =\frac{1}{\pi} \int_{0}^{\pi} \mathscr{T}_{\alpha, l}^{-1}\left\{(\cosh (\beta)-\sinh (\beta) \cos (t))^{\left.-1 / 2+\sqrt{1 / 4+n^{2 \alpha}}\right\}} d t,\right. \\
& \mathscr{T}_{\alpha, l}^{-1}\left\{p_{-1 / 2+\sqrt{1 / 4+n^{2 \alpha}+\alpha n^{2 \alpha-1}}}(\cosh (\beta)) ; n \rightarrow x\right\} \\
& =\frac{1}{\pi} \int_{0}^{\pi} \mathscr{T}_{\alpha, l}^{-1}\left\{(\cosh (\beta)-\sinh (\beta) \cos (t))^{-1 / 2+\sqrt{1 / 4+n^{2 \alpha}+\alpha n^{2 \alpha-1}}}\right\} d t .
\end{aligned}
$$

For the case $1<\alpha<2$, we can use the associated integral representations as the third and fourth approximations.

\section{Concluding remarks}

This paper provides new inversion techniques for the fractional Legendre transforms. We applied some integral representations to get these inversions for the Dirichlet problems on sphere in terms of the gamma functions. We used the first and second approximations of the ratio of gamma functions to obtain the solution in a formal form. Also, as a complementary work we can survey other approximations of asymptotic expansion to extend the solution of fractional Legendre differential equation (1-4) for $m-1<\alpha \leq m, m=3,4, \ldots$.

Acknowledgment. The author wishes to thank the unknown referee for constructive comments. Also, he would like to express his sincere appreciation to the Shahrekord university and the center of excellence for mathematics for financial supports.

\section{REFERENCES}

[1] M. Abramowitz AND I. A. Stegun, Handbook of mathematical functions with formulas, graphs, and mathematical tables, Dover, New York, 1972.

[2] A. Ansari and A. Refahi Sheikhani, New identities for the Wright and the Mittag-Leffler functions using the Laplace transform, Asian-Euro. J. Math. 7 (2014), 1450038.

[3] A. AnSARI, On finite fractional Sturm-Liouville transforms, Int. Trans. Spec. Funct. 26 (2015), 51-64.

[4] A. Ansari, M. Ahamadi Darani And M. Moradi, On fractional Mittag-Leffler operators, Rep. Math. Phys. 70 (2012), 119-131.

[5] A. Ansari, A. Refahi Sheikhani and H. Saberi Najafi, Solution to system of partial fractional differential equation using the fractional exponential operators, Math. Meth. Appl. Sci. 35 (2012), 119-123.

[6] A. ANSARI, Fractional exponential operators and time-fractional telegraph equation, Bound. Value Prob. 125 (2012). 
[7] A. Ansari, A. Refahi Sheikhani And S. Kordrostami, On the generating function $e^{x t+y \phi(t)}$ and its fractional calculus, Cent. Euro. J. Phys. 11 (2013), 1457-1462.

[8] E. BAs, Fundamental spectral theory of fractional singular Sturm-Liouville operator, J. Funct. Spac. Appl. 2013 (2013), Article ID 915830.

[9] A. V. Bobylev and C. Cercignani, The inverse laplace transform of some analytic functions with an application to the eternal solutions of the Boltzmann equation, Appl. Math. Lett. 15 (2002), 807-813.

[10] R. V. Churchill, The operational calculus of Legendre transforms, J. Math. Phys. 33 (1954), $165-178$.

[11] R. V. Churchill and C. L. Dolph, Inverse transforms of products of Legendre transforms, Proceed. Amer. Math. Soc. 5 (1954), 93-100.

[12] R. V. Churchill, New operational mathematics: the operational calculus of Legendre Transforms, Technical report no. 1, project 2137 ordnance corps, US army, contract no. DA-20-018-ORD-12916, August, 1953.

[13] E. T. Copson, Asymptotic expansions, Cambridge University Press, London, 1965.

[14] L. Debnath and D. Bhatta, Integral transforms and their applications, 2nd ed., Chapman \& Hall/CRC, Taylor \& Francis Group, New York, 2007.

[15] A. Erdelyi, W. Magnus, F. Oberhettinger and F. G. Tricomi, Higher transcendental functions, I, McGraw-Hill, New York, 1953.

[16] A. A. Kilbas, H. M. Srivastava and J. J. Trujillo, Theory and applications of fractional differential equations, Elsevier, Amsterdam, 2006.

[17] M. Klimek and O. P. Agrawai, On a regular fractional Sturm-Liouville problem with derivatives of order in $(0,1)$, Proceedings of the 13th International Carpathian Control Conference, Vysoke Tatry (Podbanske), Slovakia, 2012, dx.doi.org/10.1109/CarpathianCC. 2012.6228655 .

[18] M. Klimek and O. P. Agrawal, Fractional Sturm-Liouville problem, Comput. Math. Appl. 66 (2013), 795-812.

[19] N. N. Lebedev, Y. S. Uflyand and I. P. SKalskaya, Worked problems in applied mathematics, Dover, New York, 1965.

[20] F. MAINARDI, Fractional calculus and waves in linear viscoelasticity, Imperial College Press, London, 2010.

[21] F. Oberhettinger, Tables of Mellin transforms, Springer, New York, 1974.

[22] G. PAGninI, The M-Wright function as a generalization of the Gaussian density for fractional diffusion processes, Fract. Calc. Appl. Anal. 16 (2013), 436-453.

[23] M. Rivero, J. J. Trujillo and M. P. Velasco, A fractional approach to the Sturm-Liouville problem, Cent. Euro. J. Phys. 11 (2013), 1246-1254

[24] I. Podlubny, Fractional differential equations, Academic Press, San Diego, 1999.

[25] I. N. Sneddon, The use of integral transforms, Mac Graw-Hill, New York, 1979.

[26] C. J. Tranter, Legendre transforms, Quart. J. Math. Oxford J. 2 (1950), 1-8.

\author{
Alireza Ansari \\ Department of Applied Mathematics \\ Faculty of Mathematical Sciences \\ SHAHREKORD UNIVERSITY \\ P. O. Box 115, SHAHREKORD \\ IRAN \\ E-mail: alireza_1038@yahoo.com
}

